\title{
Avaliação de parâmetros de qualidade e efeito do congelamento na conservação de polpas de butiá
}

\author{
Evaluation of quality parameters and the effect of freezing \\ on the conservation of butiá pulp
}

Lia Raquel Ciervo de Oliveira ${ }^{1}$

Adriana Aparecida Hansel Michelotti2

Denise Filippin de Lima Rocha ${ }^{3}$

Melissa Walter ${ }^{4}$

Joseana Severo 5
RECEBIDO EM 13/04/2021 ACEITO EM 16/06/2021

\begin{abstract}
RESUMO
Entre as frutas nativas do Rio Grande do Sul, o butiá se destaca, sendo amplamente utilizado em agroindústrias e na culinária regional. Com o objetivo de avaliar as características de qualidade de polpas de butiá produzidas por produtores integrantes da Cadeia Solidária das Frutas Nativas, foram realizadas análises em duas etapas. Na primeira etapa, seis amostras foram avaliadas; na segunda etapa, duas amostras de polpa de butiá foram congeladas e analisadas pelo período de 60 dias. Realizaram-se análises físico-químicas no dia do despolpe e após 30 e 60 dias; realizaram-se análises microbiológicas após 30 e 60 dias de armazenamento $\mathrm{a}-20{ }^{\circ} \mathrm{C}$. No primeiro experimento, as 6 polpas demostraram uma variação no teor de sólidos solúveis totais (SST), estando todos os
\end{abstract}

\footnotetext{
1 Especialista pelo Instituto Federal Farroupilha, IFFar, Campus Santa Rosa, Rio Grande do Sul, Brasil. liaciervo@yahoo.com.br - https://orcid.org/0000-0002-8933-9232

2 Professora Mestre no Instituto Federal Farroupilha, IFFar, Campus Santa Rosa, Rio Grande do Sul, Brasil. adriana.michelotti@iffarroupilha.edu.br - https://orcid.org/0000-0002-7537-0140

3 Professora Mestre no Instituto Federal Farroupilha, IFFar, Campus Santo Augusto, Rio Grande do Sul, Brasil. denise.rocha@iffarroupilha.edu.br - https://orcid.org/0000-0001-6758-3798

4 Professora Doutora no Instituto Federal Farroupilha, IFFar, Campus Santa Rosa, Rio Grande do Sul, Brasil. melissa.walter@iffarroupilha.edu.br - https://orcid.org/0000-0003-0535-0352

5 Professora Doutora no Instituto Federal Farroupilha, IFFar, Campus Santa Rosa, Rio Grande do Sul, Brasil. joseana.severo@iffarroupilha.edu.br - https://orcid.org/0000-0003-0571-1955
} 
valores obtidos de acordo com a legislação. Quanto à acidez total titulável (ATT), 3 amostras apresentaram teores abaixo do valor mínimo fixado. Os teores de fenóis totais das amostras variaram de 865,9 mgEAC.100 g-1 a 1239,3 mgEAC.100g $\mathrm{g}^{-1}$. Quanto à presença de carotenoides, os teores variaram entre 28,6 $\mu \mathrm{gEKcar} . \mathrm{g}^{-1} \mathrm{e}$ 53,9 $\mu \mathrm{gE} \beta$ car.g-1. Houve diminuição dos compostos bioativos durante o armazenamento das polpas; além disso, as contagens microbiológicas revelaram que, após 60 dias, uma das amostras apresentou contagens superiores, reforçando a importância das Boas Práticas de Fabricação (BPF). Dessa forma, verifica-se que há variação nas características físico-químicas e de compostos bioativos entre as polpas de butiá congeladas analisadas e que o armazenamento teve influência negativa na manutenção dos compostos fenólicos, sem efeito significativo para os carotenoides.

PALAVRAS-CHAVE: armazenamento; fenóis; carotenoides; atividade antioxidante.

\section{ABSTRACT}

Butiá is a native fruit of Rio Grande do Sul that stands out due to the associated traditional value, peculiar flavor, and aroma, being widely used in the preparation of various culinary dishes. The present work aimed to evaluate the quality characteristics of frozen butiá pulps produced by farmers who are part of the solidarity network of native fruits. The research was carried out in two stages, in the first six samples were collected from farmers who produce pulp for commercialization. In the second stage, a sample of frozen pulp, from a farmer, was compared with a pulp produced in the food laboratory of the Federal Farroupilha Institute, during the 60 days. Physical-chemical analyzes were carried out on the day of the pulping and after 30 and 60 days of storage at $-20^{\circ} \mathrm{C}$, while the microbiological analyzes were carried out in the period of 30 and 60 days. There was variation in the parameters evaluated. All pulps showed a variation in the content of total soluble solids (SST), between 8.6 and $14.6^{\circ}$ Brix, being within the standards of IN 37/2018. As for total titratable acidity (ATT), 3 samples had levels below $0.8 \%$, the minimum value set in the Identity and Quality Standard (PIQ), for this parameter. The total phenol content of the samples ranged from 1896.6 mgEAG.100 $\mathrm{g}^{-1}$ to 2444 mgEAG.100 $\mathrm{g}^{-1}$. Total carotenoids varied between $28.6 \mu \mathrm{gE} \beta c a r . g^{-1}$ and $53.9 \mu \mathrm{gE \beta car} . \mathrm{g}^{-1}$. When the storage effect was evaluated, both samples showed similar behavior in their contents, as expected, the ATT increased with the storage time and the bioactive compounds decreased. In addition, microbiological counts revealed that after 60 days the IFFar pulp was following the law. However, the pulp from the producer had a higher count than the IFFar pulp, reinforcing the importance of 
Good Manufacturing Practices (GMP). Thus, it appears that there is variation in the physical-chemical characteristics and bioactive compounds between samples obtained from different producers, in addition, it was found that storage had a negative influence on the maintenance of bioactive compounds, however, the degradation was not significant for carotenoids.

KEYWORDS: storage; phenols; carotenoids; antioxidant activity.

\section{Introdução}

O butiá, assim como o araçá, a guabiroba, a jabuticaba e a pitanga destacam-se entre as diversas espécies de frutas nativas presentes no sul do Brasil. O interesse por espécies nativas vem crescendo, devido aos benefícios que o consumo pode propiciar à saúde humana (SGANZERLA, 2010), além de serem alternativas para a geração de renda em pequenas propriedades rurais. O uso tradicional dos frutos e folhas do butiazeiro para a elaboração de produtos fortalece e valoriza os grupos de agricultores familiares, através de ações que apoiam o uso e o manejo sustentável das frutas nativas (EMBRAPA, 2004; SEVERO, 2020).

A partir de levantamentos de campo e herbários no Rio Grande do Sul são relatadas oito espécies de butiá como nativas, são elas: Butia exilata Deble \& Marchiori; B. lallemantii Deble \& Marchiori, B. catarinenses Noblick \& Lorenzi, B. eriospatha (Mart ex Drude) Becc., B. odorata (Barb. Rodr.) Noblick, B. paraguayensis (Barb. Rodr.) L. H. Bailey, B. witeckii K. Soares \& S Longhi e B. yatay (Mart.) Becc. O Estado registra a segunda maior diversidade de espécies de butiá do Brasil, estando atrás apenas do Mato Grosso do Sul (SOARES, 2013, p. 3).

O butiá pode ser consumido através do fruto in natura ou usado na elaboração de pratos culinários, doces e bebidas, muitos dos quais são preparados a partir da polpa do fruto previamente obtida e congelada, processo que possibilita a oferta contínua do produto ao longo do ano. A Instrução Normativa (IN) Nº1, de 7 de janeiro de 2000, do Ministério da Agricultura e Pecuária 
(MAPA), define polpa de fruta como sendo o "produto não fermentado, não concentrado, não diluído, obtido de frutos polposos, por meio de processo tecnológico adequado, com um teor mínimo de sólidos totais, proveniente da parte comestível do fruto" (BRASIL, 2000). Já na IN N³7, de 01 de outubro de 2018, o MAPA fixou os padrões de identidade e qualidade (PIQ) para o suco/polpa de butiá, cujo valor mínimo para sólidos solúveis deve ser de $6{ }^{\circ}$ Brix, a $20^{\circ} \mathrm{C}$; pH 2, no mínimo; e acidez total, expressa em ácido cítrico, de 0,8\%, no mínimo (BRASIL, 2018).

A industrialização de polpas visa à transformação da matéria in natura em produtos com características microbiológicas e sensoriais seguras. Entretanto, sem os cuidados de Boas Práticas de Fabricação (BPF) é inevitável que ocorram transformações químicas, bioquímicas e microbiológicas nesses produtos. A qualidade da fruta, assim como da polpa, está relacionada a fatores envolvidos na fase de pré-colheita e de pós-colheita (LANZ et al. 2019, p. 96).

A microbiota presente em frutos constitui-se de bolores, leveduras, bactérias lácticas e outros microrganismos ácidos tolerantes (SIQUEIRA; BORGES, 1997 apud SANTOS; COELHO; CARREIRO, 2008, p. 913).

A microbiota deteriorante da polpa pode ser proveniente de microrganismos presentes naturalmente na fruta, ou pode ser introduzida pela ruptura da casca, falta de sanitização da matéria-prima e/ou condições higiênicas insatisfatórias dos manipuladores e das instalações industriais, que permitem a proliferação (CABRAL; JÚNIOR FREIRE; MATTA, 2010; CENCI, 2006 apud LANZ et al. 2019, p. 96).

A acidez presente no butiá, auxilia no controle do crescimento de alguns grupos de microrganismos, inclusive alguns patogênicos. No entanto, é importante que existam parâmetros microbiológicos para esses produtos, tendo em vista que são passíveis de contaminações.

A IN N $01 / 2000$, de 07 de janeiro de 2000, do MAPA, estabelece que as características físicas, químicas e organolépticas deverão ser as provenientes do fruto de sua origem, através dos Parâmetros de Identidade e Qualidade (PIQ’s), observando os limites mínimos e máximos fixados para cada polpa de fruta. Em relação às características microbiológicas, fixa o limite máximo 
de $5 \times 10^{-3} \mathrm{UFC} / \mathrm{g}^{-1}$ para contagem de bolores e leveduras em polpas de frutas in natura, não estabelecendo padrão para bactérias mesófilas (BRASIL, 2000 apud Lanz et al. 2019).

A fundamentação científica a respeito dos benefícios do consumo do butiá e o estudo para a obtenção de um produto final de qualidade são instrumentos valiosos a serem aliados às dinâmicas já existentes. Visa-se a estimular a conservação e a manutenção da biodiversidade nativa, a geração de emprego e renda, a cooperação e a promoção da soberania alimentar, com sintonia entre campo e cidade (LONGHI, 2014).

Estudos indicam a presença de carotenoides e compostos fenólicos no Butiá, compostos que podem atribuir propriedades funcionais ao fruto, já que trazem benefícios como a redução do risco de doenças crônicas não transmissíveis (SGANZERLA, 2010; ROCKETT et al., 2020). Alguns carotenoides, como o $\beta$-caroteno, são fontes de vitamina A, que age na manutenção da visão, na integridade do sistema imunológico, na diferenciação e proliferação celular e na prevenção de doenças crônico-degenerativas (VAN JAARSVELD et al., 2006). Além de fornecer bioativos, o butiá é uma fonte promissora de antioxidantes naturais, com potencial para aplicação em alimentos e/ou produtos farmacêuticos, incentivando e valorizando o uso sustentável da biodiversidade (ROCKETT et al., 2020).

O presente estudo tem como objetivo avaliar as características físico-químicas de polpas de butiá congeladas provenientes de 6 produtores integrantes da Cadeia Solidária das Frutas Nativas no Noroeste do Rio Grande do Sul e avaliar o efeito do armazenamento a - $20^{\circ} \mathrm{C}$, por 60 dias, na preservação das suas características físico-químicas e microbiológicas. 


\section{Materiais e Métodos}

\subsection{Experimento 1}

Para a primeira etapa da presente pesquisa, analisaram-se polpas de butiá congeladas provenientes de 6 produtores participantes da Cadeia Solidária das Frutas Nativas, de diferentes localidades da região Noroeste do Rio Grande do Sul: Porto Vera Cruz, Tucunduva, Três de Maio e Giruá. As amostras foram coletadas com os produtores, já congeladas, em agosto de 2018, e imediatamente encaminhadas para o Laboratório de Tecnologia de Alimentos do IFFar, Campus Santa Rosa, sendo mantidas sob congelamento $\left(-20^{\circ} \mathrm{C} \pm 2^{\circ} \mathrm{C}\right)$ até a realização das análises.

\subsection{Experimento 2}

Para a segunda etapa da pesquisa, foram utilizadas duas amostras de polpa, uma fornecida por uma produtora da cidade de Giruá (amostra Produtora) e outra produzida no Laboratório de Tecnologia de Alimentos do IFFar, Campus Santa Rosa, elaborada a partir de frutos doados pela mesma produtora (amostra IFFar). Os frutos foram retirados do cacho e selecionados visualmente quanto à aparência, cor e ausência de danos físicos. Depois, foram separados da corola e sanitizados em solução de hipoclorito de sódio a 2\%, por 15 minutos. Foram, então, enxaguados em água corrente e submetidos ao despolpe em uma despolpadeira previamente limpa e sanitizada com solução de hipoclorito de sódio a $2 \%$. A polpa extraída foi fracionada e acondicionada em 12 sacos plásticos, cada um contendo 200 g, e, posteriormente, congelada em freezer no Laboratório de Tecnologia de Alimentos do IFFar. Foram realizadas as análises físico-químicas nos tempos 0, 30 e 60 dias de armazenamento congelado $\left(-20^{\circ} \mathrm{C} \pm 2{ }^{\circ} \mathrm{C}\right)$ e análises microbiológicas após 30 e 60 dias de armazenamento. 


\subsection{Análises}

\subsubsection{Determinação de sólidos solúveis totais (SST)}

O teor de SST foi determinado por refratometria, utilizando-se refratômetro de mesa, marca Shimatzu ${ }^{\oplus}$, de acordo com as normas do Instituto Adolfo Lutz (IAL, 2008). Os resultados foram expressos em ${ }^{\circ}$ Brix.

\subsubsection{Determinação de acidez total titulável (ATT)}

A acidez ATT foi determinada pelo método da titulação volumétrica com indicador, conforme as normas do Instituto Adolfo Lutz (IAL, 2008). Os resultados foram expressos em \% de ácido cítrico.

\subsubsection{Relação SST/ATT (ratio)}

A relação SST/ATT foi obtida pela relação direta dos valores de sólidos solúveis totais e acidez total titulável.

\subsubsection{Carotenoides totais}

Para determinar o teor de carotenoides totais, foi utilizado o método espectrofotométrico, proposto por Rodriguez-Amaya (1999), com leitura realizada a $450 \mathrm{~nm}$, com resultados expressos em $\mu \mathrm{gE} \beta$-caroteno $\mathrm{g}^{-1}$.

\subsubsection{Cálculo do valor de provitamina A}

Para calcular o valor provitamina A foi adotado o fator de conversão fornecido pela IN No 269/2005, de 23 de setembro de 2005, em que 1 ugE $\beta$ -

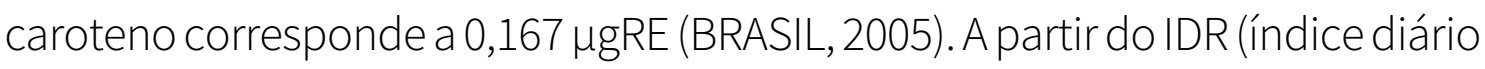
recomendado) de 600 MgRE necessários para um adulto, foram realizados os cálculos dos percentuais de 15 \% e 30 \% para se obter os parâmetros mínimos segundo os quais o produto pode ser classificado como fonte ou como alto conteúdo, a Agencia Nacional de Vigilância Sanitária (ANVISA), 
através da Resolução da Diretoria Colegiada (RDC) N 54, de 12 de novembro de 2012 (BRASIL, 2012). Dessa forma, uma porção de $100 \mathrm{~g}$ de polpa de butiá precisa ter no mínimo 538,9 $\mu \mathrm{gE} \beta$-caroteno.100-1 para ser considerada fonte de vitamina A, e no mínimo 1777,8 $\mu \mathrm{gE} \beta$-caroteno.100-1 para ser considerada um produto de alto conteúdo de vitamina $\mathrm{A}$.

\subsubsection{Compostos fenólicos totais}

O teor de compostos fenólicos totais foi determinado por meio da adaptação do método Folin-Ciocalteu (SINGLETON E ROSSI, 1965). Um grama da polpa de butiá foi diluído em $60 \mathrm{~mL}$ de água destilada, adicionando-se, em seguida, $5 \mathrm{~mL}$ do reagente folin-ciocalteau 2N (Sigma ${ }^{\circledR}$ ). Aguardou-se 20 minutos, e foram adicionados $40 \mathrm{~mL}$ de solução de carbonato de sódio a $20 \%$, completando-se o volume de 100 mL com água destilada. Após 2 horas de reação no escuro, foram realizadas as leituras em espectrofotômetro a $725 \mathrm{~nm}$. Os resultados foram expressos em mgEAC.100 g-1 (equivalente ácido clorogênico por 100 g de amostra), a partir da construção de curva-padrão com ácido clorogênico.

\subsubsection{Atividade antioxidante}

A atividade antioxidante foi determinada pelo método espectrofotométrico DPPH (2,2difenil1picrilhidrazila), e os resultados expressos em \% de inibição do DPPH, por meio de adaptação da metodologia proposta por Brand-Willians et al. (1995).

\subsubsection{Determinação de bolores e leveduras}

A contagem de bolores e leveduras foi determinada a partir do método de plaqueamento direto em superfície das diluições $10^{-1}$, $10^{-2}$ e $10^{-3}$ em meio Ágar Batata Dextrose (BDA) acidificado. Alíquotas de $100 \mu \mathrm{L}$ foram semeadas na superfície dos meios e homogeneizadas com o auxílio de uma alça de 
Drigalsky. Em seguida, as placas foram incubadas a $25^{\circ} \mathrm{C}$ por um período de 3 a 5 dias. Os resultados foram expressos pelo número de Unidades Formadoras de Colônia por grama de polpa (UFC/g).

\subsubsection{Determinação de bactérias mesófilas aeróbias}

A contagem de bactérias mesófilas foi determinada pelo método de plaqueamento em profundidade das diluições $10^{-1}, 10^{-2}$ e $10^{-3}$ em meio Plate Count Agar (PCA). Alíquotas de $1000 \mu \mathrm{L}$ foram colocadas sobre a superfície de placas, e, em seguida, o meio fundido foi vertido sobre a cultura, homogeneizando-se suavemente com movimentos circulares. Em seguida, as placas foram incubadas em posição invertida a $36^{\circ} \mathrm{C}$ por 48 horas. Os resultados foram expressos como UFC/g de polpa.

\subsubsection{Análise Estatística}

As análises foram realizadas em triplicata, e as médias obtidas foram utilizadas para cálculo do desvio padrão e submetidas à ANOVA e ao teste de Fisher a 5 \% de significância (XLSTAT, 2015).

Para determinar a correlação entre os componentes bioativos e a atividade antioxidante, foi aplicada a correlação de Pearson, utilizando-se o Excel.

\section{RESULTADOS E DISCUSSÃO}

\subsection{Experimento 1}

Na figura 1 estão apresentados os resultados obtidos nas análises das polpas coletadas com seis produtores integrantes da cadeia solidária de frutas nativas do Noroeste do Rio Grande do Sul. 
FIGURA 1 - Teores de SST (A), ATT (B), SST/ATT (C), Fenóis Totais (D), Carotenoides Totais

(E) e Atividade Antioxidante (F) em polpas de Butiá de diferentes produtores da cadeia solidária das frutas nativas.

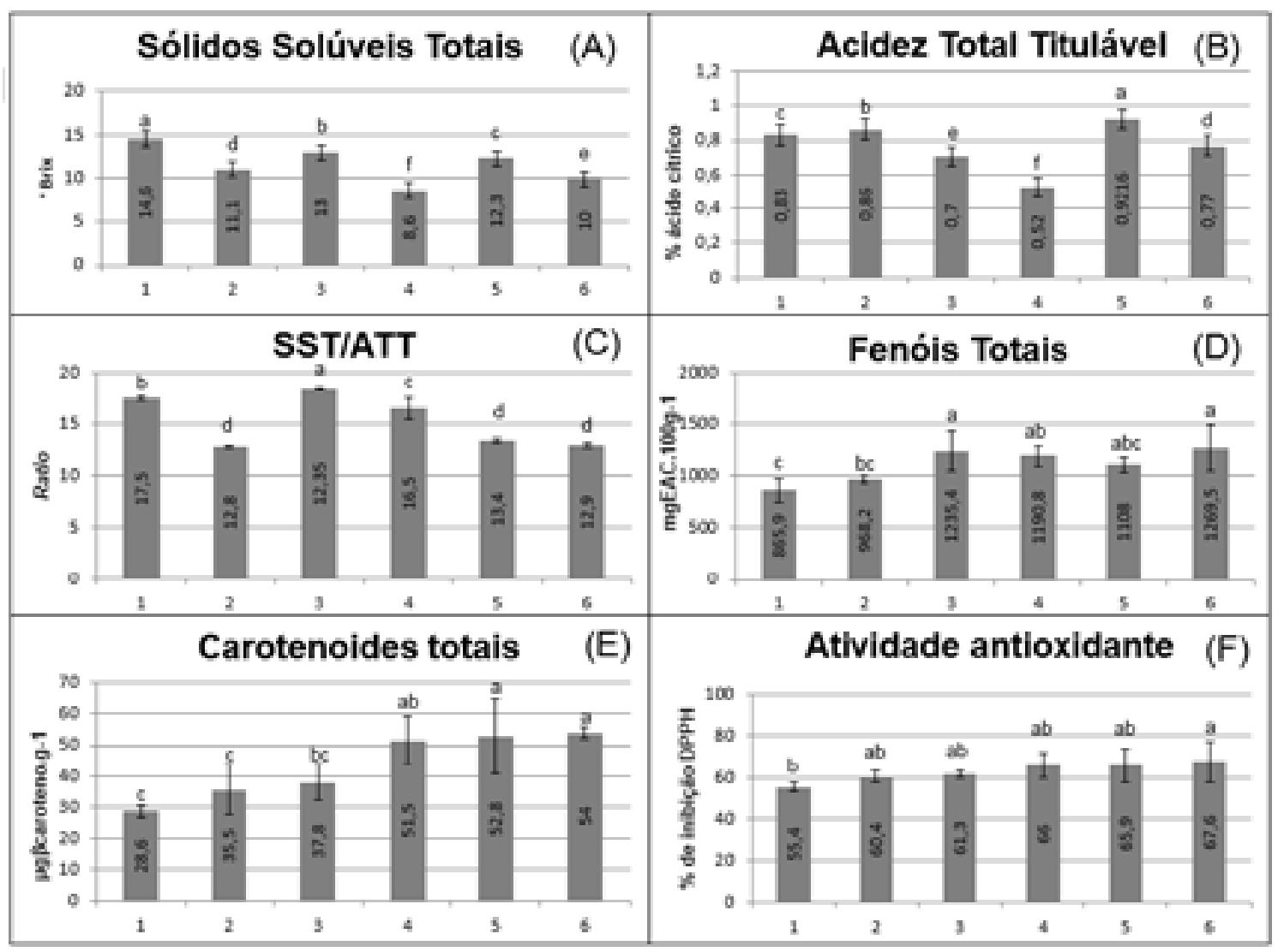

*Letras sobrescritas: letras minúsculas iguais nas colunas indicam que não há diferenças significativas entre as polpas de diferentes amostras analisadas, de acordo com o teste de Fisher a 5\% de significância.

Fonte: elaborado pelos próprios autores.

Observa-se que os índices dos parâmetros avaliados variaram entre as polpas, resultado que pode ser decorrente de diferenças na forma de extração da polpa, da maturação dos frutos e das espécies de butiás utilizadas no preparo. O fruto do butiazeiro tem características físico-químicas distintas mesmo entre populações de uma mesma espécie. No estudo da composição química de frutos de butiazeiro do gênero B. odorata Becc., Fonseca (2012), verificou que os teores de sólidos solúveis totais (SST), pH, acidez total (ATT) e teor de açúcar variaram significativamente entre os frutos de quatro butiazeiros. Dal Magro et. al. (2006) compararam a composição físico-química de butiás do gênero B. eriospatha e também relatam variações. Schwartz et. al. 
(2009) verificaram que as propriedades e/ou as variações genéticas entre três populações de B. capitata, do município de Santa Vitória do Palmar (RS), apresentaram variabilidade em teores de SST e ATT, volume do suco produzido, coloração de epiderme, entre outros.

Os teores de SST (Figura 1. A) encontrados em todas as amostras analisadas estão acima do valor mínimo, que é de $6^{\circ}$ Brix, conforme regulamentação da IN No 37/2018, variando entre 8,6 e 14,6 Brix e apresentando diferença significativa entre todas as amostras. Martins et al. (2019) relataram teores de 11,5 e $12,7^{\circ}$ Brix para fruto e polpa de B. yatay, respectivamente. Fonseca (2012) encontrou diferença significativa nos teores de SST determinados em 4 amostras B. odorata Barb. Rodr., com valores variando entre 10,6 e 14,8 ${ }^{\circ}$ Brix.

Quanto aos teores de ATT (Figura 1. B), observou-se variação significativa entre as seis amostras, com valores entre 0,5 e 0,9 \%; ainda, que 3 amostras apresentaram teores abaixo de 0,8\%, valor mínimo fixado no PIQ para esse parâmetro (BRASIL, 2018). Martins et al. (2019) encontraram teores de 1,3 e 1,5\% de ácido cítrico no fruto e polpa de B. yatay, respectivamente. Fonseca (2012) determinou teores de ATT entre 1,74 \% e 3,47 \% para B. odorata Barb. Rodr, enquanto SGANZERLA (2010) relatou teores de 1,92 \% e 1,88\%, para B.capitata e B. eriosphata, respectivamente.

A relação SST/ATT (Figura 1. C) variou entre 12,8 e 18,35, no entanto não se alterou significativamente entre as amostras 2, 5 e 6; a média entre as amostras foi de 13,6. A relação SST/ATT indica o grau de equilíbrio entre a acidez e os teores de açúcar do fruto. Quanto maior a maturação do fruto, maior é a concentração de açúcar e menor é o teor de acidez (CHITARRA, CHITARRA, 2005). A legislação não determina valores para essa relação, mas ao adotarmos os valores para o padrão de identidade e qualidade de polpa de butiá $6^{\circ}$ Brix e 0,8 \% de acidez, teremos o ratio 7,5. Dessa forma, observa-se que os valores encontrados para as 6 polpas de butiá avaliadas estão acima de 7,5. 
Os maiores teores de fenóis totais (Figura 1. D) foram identificados nas polpas 3, 4, 5 e 6, e não apresentaram diferença significativa entre si. Os valores encontrados no presente estudo variaram de 865,9 mgEAC.100 ${ }^{-1}$ a 1235,4 mgEAC.100g-1, inferiores aos relatados nos estudos de Fonseca

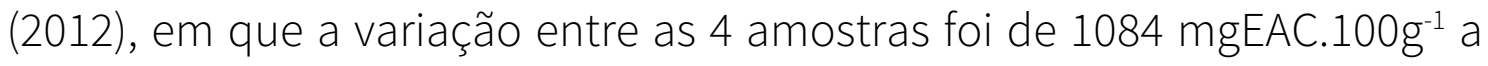
1571,9 mgEAC.100g-1 , e superiores aos valores encontrados por Araújo et al. (2017), de 636 mgEAC.100 $\mathrm{g}^{-1}$.

Hoffmann (2016) identificou 23 compostos fenólicos nas espécies de butiá avaliadas em seu trabalho.

O ácido clorogênico foi o ácido fenólico majoritário em todas as espécies. Ele representou 35, 38, 37 e 55\% do total de ácidos fenólicos nas espécies B. catarinensis, B. odorata, B. paraguayensis e B. yatay, respectivamente. O teor de ácido clorogênico variou de 9,8 (B. catarinensis) a 18,8 $\mu \mathrm{g} \cdot \mathrm{g}^{-1}$ (B. yatay). O teor de ácido clorogênico em frutos de butiá é similar ao encontrado em maçãs red delicious (11,0 $\left.\mu \mathrm{g} \cdot \mathrm{g}^{-1}\right)$, melão $\left(5,9 \mu \mathrm{g} \cdot \mathrm{g}^{-1}\right)$, carambolas $\left(6,10 \mu \mathrm{g} \cdot \mathrm{g}^{-1}\right)$, pera $\left(7,2 \mu \mathrm{g} \mathrm{g} \mathrm{g}^{-1}\right)$ e abacaxi $\left(13,10 \mu \mathrm{g} \cdot \mathrm{g}^{-1}\right)$ (FU et al., 2011 apud HOFFMANN, 2016 p. 57).

Na quantificação dos carotenoides (Figura 1. E), as polpas 4, 5 e 6 foram as que apresentaram os maiores teores, 52,7 $\mu \mathrm{gE} \beta \mathrm{car} . \mathrm{g}^{-1}$, em média, sem evidência de diferença significativa entre elas, superiores, portanto, aos 39,77 $\mu \mathrm{g} \cdot \mathrm{g}^{-1}$ relatados por Pereira et al. (2013). Os menores teores foram observados nas amostras 1, 2 e 3, que não apresentaram diferença significativa, com média de 28,6 $\mu \mathrm{gE} \beta \mathrm{car}_{\mathrm{g}} \mathrm{g}^{-1}$, resultados superiores aos encontrados por Araújo et al. (2017), que obtiveram 24,10 $\mu g E \beta c a r . g^{-1}$ para o butiá.

Pereira et al. (2013), identificaram o $\beta$-caroteno $(21,67 \mu \mathrm{g} / \mathrm{g})$ como majoritário em frutos de butiá (B. capitata), representando mais de 50\% do seu conteúdo (39,77 g/g). Além disso, o isômero 9cisß-caroteno foi identificado em quantidades consideráveis $(10,17 \mu \mathrm{g} / \mathrm{g})$, representando $25,6 \%$ do conteúdo. A luteína representou $11,8 \%(4,68 \mu \mathrm{g} / \mathrm{g})$, seguida de pequenas quantidades do isômero 13cis $\beta$-caroteno, 5,6epoxy $\beta$-caroteno, criptoxantina e zeaxanthina. 
Verifica-se que a concentração de $\beta$-caroteno encontrada no butiá é superior à relatada em outras frutas, como cajá, caju, laranja, maracujá, manga, melancia, melão, pêssego e pitanga, e, também, em alguns vegetais, como o brócolis, e semelhante aos teores encontrados em cenouras (RODRIGUES-AMAYA et al., 2008).

Considerando a média entre todas as amostras de carotenoides, de 43,36 $\mu \mathrm{gE} \beta \mathrm{car}_{\mathrm{g}} \mathrm{g}^{-1}$, podemos afirmar que a média de carotenoides em uma porção de 100 gramas de amostra é de 4336,6 $\mu \mathrm{gE} \beta$ car, que, conforme a RDC N54/2012, torna o produto apto a ser declarado "de alto conteúdo" de carotenoides, já que contém mais de 30 \% da IDR fixada pela IN N²69/2005 (BRASIL, 2005).

Entre os fatores que afetam a composição dos carotenoides nos frutos, estão: espécie, variedade, parte do vegetal, grau de maturação, clima, tipo de solo, condições de cultivo e colheita, processamento e armazenamento (SGANZERLA, 2010; FONSECA, 2012; HOFFMANN et al. 2014).

A atividade antioxidante (figura 1 . F) variou significativamente entre as amostras 1 e 6, que apresentaram a menor e a maior atividade antioxidante, 55 \% e 67 \% de inibição, respectivamente. Houve correlação positiva entre os carotenoides e a atividade antioxidante $(0,9816)$, assim como entre os fenóis e a atividade antioxidante $(0,8647)$. Santos et al. (2010) observaram que houve correlação entre a presença de compostos bioativos e atividade antioxidante em polpas de cupuaçu.

A variação observada entre os resultados obtidos nos parâmetros avaliados nas polpas de butiá também pode ser relacionada com a forma de processamento adotada pelos integrantes da Cadeia Solidária das Frutas Nativas, que utilizam frutos obtidos a partir de diversas espécies de butiá. A despolpadeira é usada coletivamente, circulando entre as comunidades, o que pode dificultar também o padrão de higiene das polpas obtidas. 


\subsection{Experimento 2}

Na tabela 1 estão apresentados os resultados referentes às análises das duas polpas realizadas durante 60 dias de armazenamento.

TABELA 1 - Características físico-químicas da polpa de butiá congelada e armazenada durante 60 dias.

\begin{tabular}{|c|c|c|c|c|c|c|}
\hline \multicolumn{7}{|c|}{ COMPOSIÇÃO FÍSICO-QUÍMICA } \\
\hline \multirow[t]{2}{*}{$\begin{array}{c}\text { Tempo de } \\
\text { armazenamento } \\
\text { (dias) }\end{array}$} & \multicolumn{2}{|c|}{$\begin{array}{l}\text { Sólidos Solúveis Totais } \\
\qquad\left({ }^{\circ} \text { Brix }\right)\end{array}$} & \multicolumn{2}{|c|}{$\begin{array}{l}\text { Acidez Total Titulável } \\
\text { (\% ácido cítrico) }\end{array}$} & \multicolumn{2}{|c|}{$\begin{array}{c}\text { Sólidos Solúveis Totais/ } \\
\text { Acidez Total Titulável } \\
\text { (ratio) }\end{array}$} \\
\hline & Produtor & IFFar & Produtor & IFFar & Produtor & IFFar \\
\hline 0 & $9,5 \mathrm{aA}$ & $9,5 \mathrm{aA}$ & $0,67 \mathrm{aB}$ & $0,84 \mathrm{aA}$ & $14,8 \mathrm{aA}$ & $11,25 \mathrm{aB}$ \\
\hline 30 & $9,5 \mathrm{aA}$ & $9,5 \mathrm{aA}$ & $0,68 \mathrm{aB}$ & $0,89 \mathrm{bA}$ & $13,24 a b A$ & $11,27 \mathrm{aA}$ \\
\hline \multirow[t]{4}{*}{60} & $9,0 \mathrm{aA}$ & $9,0 \mathrm{aA}$ & $0,71 \mathrm{aB}$ & $0,88 \mathrm{bA}$ & $12,76 \mathrm{bA}$ & $11,29 \mathrm{aB}$ \\
\hline & \multicolumn{6}{|c|}{ COMPOSTOS BIOATIVOS } \\
\hline & \multicolumn{2}{|c|}{$\begin{array}{l}\text { Fenóis Totais } \\
\left(\mathrm{mgEAC} .100 \mathrm{~g}^{-1}\right)\end{array}$} & \multicolumn{2}{|c|}{$\begin{array}{l}\text { Carotenoides Totais } \\
(\mu \mathrm{gE} \beta \text {-caroteno.g-1) }\end{array}$} & \multicolumn{2}{|c|}{$\begin{array}{c}\text { Atividade Antioxidante } \\
\text { (\% inibição) }\end{array}$} \\
\hline & Produtor & IFFar & Produtor & IFFar & Produtor & IFFar \\
\hline 0 & $1259,4 \mathrm{aA}$ & $771,9 \mathrm{aB}$ & 29,4 aA & $19,8 \mathrm{aB}$ & 57,06 aA & $32,47 \mathrm{aB}$ \\
\hline 30 & $1179,82 \mathrm{abA}$ & $701,26 \mathrm{aB}$ & 29,2 aA & $17,5 \mathrm{aB}$ & $34,84 \mathrm{cA}$ & $24,39 \mathrm{bB}$ \\
\hline 60 & $1079,5 \mathrm{bA}$ & $502,77 \mathrm{bB}$ & 28,4 aA & $16,5 \mathrm{aB}$ & $46,99 \mathrm{bA}$ & $33,22 \mathrm{aB}$ \\
\hline
\end{tabular}

*Letras minúsculas nas colunas indicam diferenças significativas entre o período de armazenamento. Letras maiúsculas na linha indicam diferenças significativas entre as polpas de diferentes fontes analisadas no mesmo período de armazenamento, de acordo com o teste de Fisher a 5 \% de significância. EAC: Equivalente ácido clorogênico.

Fonte: elaborado pelos próprios autores.

Não houve alterações significativas nos teores de SST nas amostras avaliadas durante o armazenamento (Tabela 1). Também não foram verificadas diferenças significativas nos teores de SST entre a polpa da produtora e a obtida no IFFar, estando os valores de acordo com o valor mínimo estabelecido pelo PIQ para suco/polpa de butiá, que é de $6^{\circ}$ Brix (BRASIL, 2018). Hoffmann et al. (2017), avaliando o efeito do armazenamento congelado de polpa de butiá, $B$. odorata, ao longo de 12 meses, também verificaram que não houve mudança significativa nos teores de SST durante o período.

O tempo de armazenamento não alterou significativamente a ATT da polpa da produtora; no entanto, a polpa do IFFar variou significativamente 
entre o tempo 0 e os tempos 30 e 60. O teor de ATT apresentou diferença significativa entre as polpas da produtora e do IFFar em todos os períodos de armazenamento. Os teores observados na polpa da produtora foram inferiores ao valor mínimo de 0,8 \% fixado pela legislação (BRASIL, 2018). Hoffmann et al. (2017) relataram teores superiores de ATT e não observaram diferença significativa após 60 dias de armazenamento.

O conteúdo de ácidos tende a diminuir à medida que as frutas amadurecem (CHITARRA, CHITARRA, 2005), o que sugere que as frutas utilizadas na polpa do IFFar estavam menos maduras do que aquelas utilizadas para preparar a polpa do produtor. Além disso, observa-se que, somente após 30 dias de armazenamento, ela teve aumento significativo de acidez. Lanz et al. (2019) observaram que, na polpa de acerola, que também tem 0,8\% como padrão fixado pela legislação (BRASIL, 2018), houve aumento significativo nos teores de acidez após 60 dias.

O índice ratio diminuiu com o tempo de armazenamento, e a diferença só foi significativa entre os tempos 0 e 60, ou seja, apenas após 60 dias de armazenamento pôde-se observar perda de qualidade.

Os teores de fenóis diminuíram significativamente durante o armazenamento, tanto na polpa da produtora quanto na polpa do IFFar, reduzindo-se em 14,3\% e 34,9 \%, respectivamente. Quando comparadas as polpas da produtora e do IFFar, observou-se diferença significativa nos teores de fenóis, verificando-se maior presença desse composto na polpa da produtora.

Freire et al. (2013), analisando os teores de fenóis totais em diferentes polpas de frutas, durante 90 dias, relataram uma diminuição significativa de $27 \%$ nos teores de fenóis totais na polpa de acerola. Hoffmann et al. (2017), na avaliação da polpa de B. odorata, também relataram decréscimo, desta vez de $37 \%$, nos teores de compostos fenólicos após 120 dias de congelamento. A diminuição dos fenóis pode ser decorrente de diversos fatores, tendo em 
vista que se trata de um grande grupo de compostos, podendo a estabilidade variar de acordo com os fatores aos quais os frutos e as polpas são expostos. Também pode ocorrer a oxidação dos fenóis durante o armazenamento, promovida pela atividade endógena de polifenol oxidase, decorrente do processo de despolpagem (Patthamakanokporn et al., 2008).

As polpas do produtor e do IFFar não apresentaram variações significativas nos teores de carotenoides em função do tempo de armazenamento. Segundo Rodriguez-Amaya (2008), o congelamento é um método de conservação eficaz na preservação dos carotenoides nos alimentos. Assim como verificado para os compostos fenólicos, houve diferença significativa entre os níveis desses compostos detectados nas amostras: na polpa do produtor, a presença de carotenoides foi maior do que na polpa do IFFar, com média de 29 mgE $\beta c a r . g^{-1}$

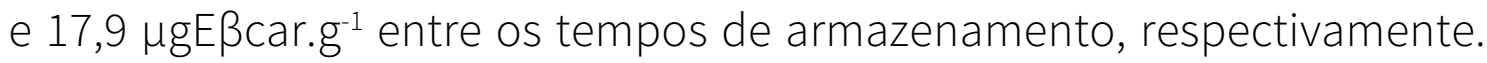
Hoffmann et al. (2017) também não encontraram diferença significativa nos teores de carotenoides no tempo 60 em relação ao tempo 0; os valores relatados

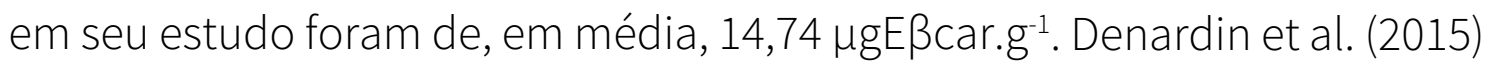
relataram teores de 3,85 $\mu \mathrm{gE} \beta \mathrm{car}_{\mathrm{g}}{ }^{-1}$ na polpa de $B$. eriosphata, valor inferior a todos os encontrados no presente estudo. A estabilidade dos carotenoides pode diferir bastante nos alimentos. "Fatores como exposição à luz e ao oxigênio, tipo de matriz alimentícia, presença de enzimas, disponibilidade de água e presença de antioxidantes e/ou pró-oxidantes podem influenciar o processo de oxidação" (RODRIGUEZ-AMAYA et al., 2008 apud Fonseca, 2012, p. 14).

A atividade antioxidante diminuiu significativamente no decorrer do tempo de armazenamento nas duas amostras, resultado esperado, já que a atividade antioxidante é influenciada pelos teores de carotenoides e de fenóis da amostra. Da mesma forma, em relação a esse parâmetro, observou-se diferença significativa entre as amostras. Hoffmann et al. (2017) também observaram 
que houve redução significativa da capacidade antioxidante da polpa de B. odorata contra o radical DPPH, que variou de 82,9 a $71,3 \%$.

Após 60 dias de armazenamento, observou-se um aumento na atividade antioxidante das polpas. Variações podem ocorrer devido à presença de outros compostos bioativos, como ácido ascórbico, além de frações individuais de compostos fenólicos e carotenoides, que também influem na atividade antioxidante (Pletsch et al., 2019). Martins et al. (2019) observaram uma redução de 44,6 \% no teor de ácido ascórbico em polpas de butiá congeladas e armazenadas durante três meses.

TABELA 2 - Contagem de bolores e leveduras e bactérias mesófilas de polpa de butiá congelada e armazenada durante 60 dias.

\begin{tabular}{ccc}
\hline $\begin{array}{c}\text { Tempo de } \\
\text { armazenamento (dias) }\end{array}$ & $\begin{array}{c}\text { Contagem de bolores e } \\
\text { leveduras (UFC/g) }\end{array}$ & $\begin{array}{c}\text { Contagem de bactérias } \\
\text { aeróbias mesófilas (UFC/g) }\end{array}$ \\
\hline 30 & $<1,0 \times 10^{1}$ & $2,0 \times 10^{2}$ \\
\hline 60 & $7,0 \times 10^{4}$ & $4,0 \times 10^{4}$ \\
\hline & IFFar & $<1,0 \times 10^{1}$ \\
\hline 60 & $<1,0 \times 10^{1}$ & $3,0 \times 10^{2}$ \\
\hline 60 & $4,0 \times 10^{3}$ & American Public Health Association $^{*}$ \\
\hline Referência & IN N $1 / 2000$ & máximo de $1 \times 10^{4} \mathrm{UFC} / \mathrm{g}^{0}$ \\
\hline
\end{tabular}

Fonte: elaborado pelos próprios autores.

A tabela 2 demonstra os resultados das análises microbiológicas. A polpa do IFFar apresentou menores contagens de bolores e leveduras e bactérias aeróbias mesófilas durante o armazenamento.

No tempo 30, ambas as polpas de butiá se apresentaram de acordo com os parâmetros definidos na legislação, que estabelece o valor máximo de $5 \times 10^{3} \mathrm{UFC} / \mathrm{g}$ (BRASIL, 2000). Após 60 dias, houve crescimento de bolores e leveduras em ambas as polpas, porém somente na polpa do produtor esse crescimento ultrapassou os valores estabelecidos pela legislação. Lanz et al. (2019) encontraram valores que variaram de $2,26 \times 10^{3}$ a $1,27 \times 10^{4}$ para 
bolores e leveduras, em polpas de acerola, após 60 dias de armazenamento, e destacaram que a contaminação pode ser atribuída à falta de sanitização antes do despolpe, diversidade de fornecedores e dificuldade na seleção da matéria-prima, devido ao seu pequeno porte.

Não há padrões oficiais definidos para microrganismos mesófilos, no entanto a American Public Health Association sugere o limite de $10^{4}$ UFC/ mL para mesófilos aeróbios (APHA, 2001). A polpa do produtor apresentou contagens maiores que o limite sugerido após 60 dias de armazenagem; por sua vez, a polpa do IFFar apresentou contagens inferiores. No estudo de Lanz et al. (2019), a contaminação por bactérias mesófilas em polpas de acerola não ultrapassou 102 UFC/g.

\section{Conclusão}

As polpas de butiá produzidas pelos integrantes da cadeia solidária das frutas nativas apresentam variação entre os produtores quanto aos parâmetros de qualidade avaliados, variação que pode ser resultado da utilização de frutos com graus diferentes de maturação, assim como de frutos de diferentes espécies e formas de extração e armazenamento.

A polpa congelada se mostra uma boa alternativa de aproveitamento dos frutos de butiá. Os compostos bioativos apresentaram estabilidade após 60 dias de armazenamento, não havendo alterações significativas no conteúdo de SST, ATT e carotenoides totais durante esse período, resultado considerado satisfatório. No entanto, faz-se necessário adotar padrões de seleção dos frutos quanto à maturação e espécie, bem como seguir as Boas Práticas de Fabricação, para garantir a qualidade do produto. É, pois, interessante aprimorar os processos utilizados, além de promover e incentivar o consumo da polpa do butiá, que apresenta alto conteúdo de carotenoides e compostos fenólicos, cujo consumo pode trazer benefícios à saúde. 


\section{Referências}

ARAÚJO, V. F. et al. Frutas Nativas Vermelhas e Amarelas: a diversidade e suas propriedades funcionais. Revista Congrega-Urcamp, v. 1, p. 1, 2017.

AMERICAN PUBLIC HEALTH ASSOCIATION. APHA Committee on Microbiological Methods for Foods. Compendium of methods for the microbiological examination of foods. 4.ed. Washington, DC: American Public Health, 2001. 676 p.

BRASIL. Ministério da Agricultura e do Abastecimento. Instrução normativa $n^{\circ} 1$, de 7 de janeiro de 2000: regulamento técnico geral para fixação dos padrões de identidade e qualidade para polpa de frutas. 6. ed. Brasília, DF: Diário Oficial da União, 2000.

BRASIL, Ministério da Saúde, Agência Nacional de Vigilância Sanitária. Resolução RDC nº 269, de 22 de setembro de 2005: Dispõe sobre o Regulamento técnico sobre a ingestão diária recomendada (IDR) de proteína, vitaminas e minerais. Diário Oficial da República Federativa do Brasil, DF, 2005.

BRASIL. Ministério da Saúde, Agência Nacional de Vigilância Sanitária. Resolução RDC nº 54, de 12 de novembro de 2012: Dispõe sobre o Regulamento Técnico sobre informação nutricional complementar. Diário Oficial da República Federativa do Brasil Brasília, DF, 2012.

BRASIL. Ministério da Agricultura e do Abastecimento. Instrução normativa n 37, de 1 de outubro de 2018: parâmetros analíticos de suco e de polpa de frutas e listagem das frutas e demais quesitos complementares aos padrões de identidade e qualidade já fixados pelo MAPA através da IN MAPA $n^{\circ}$ 49, de 26 de setembro de 2018 para suco e polpa de frutas. 6. ed. Brasília, DF: Diário Oficial da União, 2018.

BRAND-WILLIANS, W.; CUVELIER, M.E.; BERSET, C. Use of a free radical method to evaluate antioxidant activity. Lebensmittel-Wissenschaft und Technologie/Food Science and Technology, v.28, p. 25-30, 1995.

CHITARRA, Maria Isabel Fernandes; CHITARRA, Admilson Bosco. Pós-colheita de frutos e hortaliças: fisiologia e manuseio. 2 ed. Ver, e ampl. Lavras: UFLA, 2005.

DAL MAGRO, G. N. et al. Comparação físico-química de frutos congelados de Butiá eriospatha (Mart.) Becc. do Paraná e Santa Catarina-Brasil. Revista Varia Scientia, v. 06, n. 11, p. 33-44, 2006.

DENARDIN, C. C. et al. Antioxidant capacity and bioactive compounds of four Brazilian native fruits. Journal of Food and Drug Analysis, v.23, p. 387-398, 2015.

EMBRAPA. Frutas Nativas são destaque em dois projetos de sustentabilidade da biodiversidade do RS. Brasília: Empresa Brasileira de Pesquisa Agropecuária, 2016. Disponível em: https:// embrapa.br/busca-de-noticias/-noticia/ 1230545/ frutas -nativas-sao-destaque-em-dois-projetos-de-sustentabilidade-da-biodiversidade-no-rs. Acesso em 22 de ago. de 2018.

EMBRAPA. Espécies frutíferas nativas do Sul do Brasil. Editores: Maria do Carmo Bassols Raseira et al. Pelotas: Embrapa Clima Temperado, 2004. 124 p. (Documento, 129). ISSN 1516-8840.

FONSECA, Xavier Liliane. Caracterização dos frutos de butiazeiro (Butia odorata Barb. Rodr.) Noblick \& Lorenzoni e estabilidade de seus compostos bioativos na elaboração e armazenamento de geléias. Pelotas, 2012. Dissertação (Mestrado em Ciência e Tecnologia Agroindustrial) - Faculdade de Agronomia Eliseu Maciel, Universidade Federal de Pelotas, Pelotas, 2012. 
FREIRE, J. M. et al. Quantificação de composto fenólicos e ácido ascórbico em frutas e polpas congeladas de acerola, caju, goiaba e morango. Ciência Rural, 2013, v.43, n. 12. Disponível em: http://www.scielo.br/pdf/cr/v43n12/a33213cr2012-1117.pdf. Acesso em 26 de set. 2018.

HOFFMANN, J. F.; BARBIERI, R. L.; ROMBALDI, C. V. CHAVES, F. C. Butia spp. (Arecaceae): An overview. Scientia Horticulturae, v. 179, 2014, p. 122-131.

HOFFMANN, J. F. Abordagem metabolômica para acessar características de qualidade em frutos e produtos de Butia spp. Tese de Doutorado. Universidade Federal de Pelotas (UFPEL). Pelotas - RS, 2016.

HOFFMANN, J. F. et al. Stability of bioactive compounds in butiá (Butia odorata) fruit pulp and néctar. Food chemistry, v. 237, p. 683-644, 2017.

INSTITUTO ADOLFO LUTZ - IAL. Métodos Físico-químicos para Análise de Alimentos. 4. Ed. São Paulo: IAL. Versão eletrônica, 2008.

LANZ, C. L. N.; NACHTIGAL, L.; SEVERO, J. Parâmetros de qualidade de polpas de uva e acerola congeladas. Revista Ciência e Inovação do Instituto Federal Farroupilha, v. 4, p. 94-105, 2019.

LONGHI, A. Nossas matas e nossos campos têm mais sabores, o povo mais saberes. In: SEMINÁRIO BRASILEIRO SOBRE PEQUENAS FRUTAS, 7., 2013, Vacaria. Anais... Bento Gonçalves: EMBRAPA, 2014. p. 60-64.

MARTINS, J. S. et al. Avaliação nutricional de Butiá (Butia yatai) processado. Segurança Alimentar e Nutricional, v. 26, p. 1-7, e019012, 2019.

PATTHAMAKANOKPORN, O. et al. Changes of antioxidant activity and total phenolic compounds during storage of selected fruits. Journal of food composition and analysis, v. 21, n. 3, p. 241-248, 2008.

PLETSCH, L. B. H. et al. Gelado comestível de Kefir adicionado de polpa de jabuticaba e morango. Revista Instituto Laticínios Cândido Tostes, v. 74, n. 1, p. 39-50, 2019.

PEREIRA, M. C. et al. Characterization, bioactive compounds and antioxidant potential of three Brazilian fruits. Journal of Food Composition and Analysis, v. 29, n. 1, p. 19-24, 2013.

ROCKETT, F C. et al. Phenolic compounds and antioxidant activity in vitro and in vivo of Butia and Opuntia fruits. Food Research International, v. 137, 109740, 2020.

RODRIGUEZ-AMAYA, D. B. Changes in carotenoids during processing and storage of foods. Archivos Latino Americanos de Nutrition, (1-S), 38-47, 1999.

RODRIGUEZ-AMAYA, D. B; KIMURA, M; MAYA-FARFAN, J. Fontes brasileiras de carotenoides: tabela brasileira de composição de carotenoides em alimentos. Brasília, 2008.

SANTOS, C. A. A.; COELHO, A. F. S.; CARREIRO, S. C. Avaliação microbiológica de polpas de frutas congeladas. Ciência e Tecnologia de Alimentos, v. 28, n. 4, p. 913-915, 2008.

SANTOS, G. M. et al. Atividade antioxidante e correlações com componentes bioativos de produtos comercias de cupuaçu. Ciência Rural, v. 40, n. 7, p. 1636-1642, 2010. 
SCHWARTZ, E. et al. Avaliação de populações de Butia capitata de Santa Vitória do Palmar. Revista Brasileira de Fruticultura, v. 32, n. 3, p. 736-745, 2010.

SEVERO, J. Butiá: aliando desenvolvimento, saúde e sustentabilidade. Joseana Severo, Volmir Ribeiro do Amaral, Adriana Aparecida Hansel Michelotti (org.). Santa Rosa: Instituto Federal Farroupilha Campus Santa Rosa, 2020.

SGANZERLA, Marla. Caracterização físico-química e capacidade antioxidante do butiá. Pelotas, 2010. Dissertação (Mestrado em Ciência e Tecnologia Agroindustrial) - Faculdade de Agronomia Eliseu Maciel, Universidade Federal de Pelotas, Pelotas.

SINGLETON, V. L.; ROSSI, J. A. JR. Colorimetry of total phenolic with phosphomolybdic-phosphotungstic acid reagents. American Journal of Enology and Viticulture, v. 16, p. 144-158, 1965.

SOARES, Kelen Pureza. 0 gênero Butia (Becc.) Becc. (Arecaceae) no Rio Grande do Sul com ênfase nos aspectos ecológicos e silviculturais de Butia yatay (Mart.) Becc. e Butia witeckii K. Soares \& S. Longhi. Santa Maria, 2013. Dissertação (Mestrado) - Universidade Federal de Santa Maria, Santa Maria, 2013.

Van Jaarsveld, P. J. et al. Retention of $\beta$-carotene in boiled, mashed orange-fleshed sweet potato. Journal of Food Composition and Analysis, v. 19, n. 4, p. 321-329, 2006.

XLSTAT. Statistical software \& data analysis add-on for excel. Addinsoft Inc, USA, 2016. 